\title{
Magnetic properties evolution of a high permeability nanocrystalline FeCuNbSiB during thermal ageing*
}

\author{
Atef Lekdima ${ }^{\mathrm{a}}$, Laurent Morel, and Marie-Ange Raulet \\ Ampère Laboratory, UMR 5005, University of Lyon 1, 43 Bd. du 11 novembre 1918, 69100 Villeurbanne Cedex, France
}

Received: 5 December 2016 / Received in final form: 15 May 2017 / Accepted: 13 June 2017

(c) EDP Sciences 2017

\begin{abstract}
It is found to be one of the major issues while designing an aircraft, mass and volume have to be reduced in order to achieve energy efficiency. This leads to a high compactness of the electrical components which enables them to withstand at high temperatures. The magnetic components which are responsible for the electrical energy conversion, therefore exposed to high temperatures in working conditions. Their thermal ageing becomes a serious problem and deserves a particular attention. The FeCuNbSiB nanocrystalline materials have been selected for this ageing study because they are used in power electronic systems very frequently. The objective of the study is based on monitoring the magnetic characteristics under the condition of several continuous thermal ageing (100, 150, 200 and $240{ }^{\circ} \mathrm{C}$ ). An important, experimental work of magnetic characterization is being done through a specific monitoring protocol and X-ray diffraction (XRD) along with magnetostriction measurements was carried out to support the study of the evolution of the anisotropy energies with aging. The latter is discussed in this paper to explain and give the hypothesis about the aging phenomena.
\end{abstract}

\section{Introduction}

Researches are going on about the ageing of the magnetic materials irrespective of their type (polycrystalline, nanocrystalline, amorphous, ferrites, etc.). Few papers are found to be $[1,2]$ interesting on the ageing of $\mathrm{FeCuNbSiB}$ nanocrystalline materials. The main magnetic property which finds interest in these two studies is the permeability which follows a logarithmic predictive law. The finding is that the permeability decreases with ageing whatever the annealing type is (without or with a transverse magnetic field annealing). According to the reference [2], the reason relates to the induction of local anisotropy energy during ageing noted by $E_{K_{u}}^{\text {loc }}$. The latter is introduced in each magnetic domain due to a short range atomic diffusion.

In the year 1988 Yoshizawa and his team were reported the discovery of a new iron-based alloy presenting a very soft magnetic properties [3]. It is the oldest alloy of the Finemet nanocrystalline family having a composition of $\mathrm{Fe}_{\text {Ballance }} \mathrm{Cu}_{0.5-1} \mathrm{Nb}_{2-3} \mathrm{Si}_{12-16} \mathrm{~B}_{6-9}$. It was cast as an originally amorphous ribbon which was transformed to the nanocrystalline state by a subsequent annealing treatment in the temperature range of $500-600{ }^{\circ} \mathrm{C}$ during around $1 \mathrm{~h}$. The nanocrystalline alloys consist of a crystalline phase in the form of nano-grains (10-15 nm) distributed

\footnotetext{
${ }^{a}$ e-mail: atef.lekdim@gmail.com

${ }^{\star}$ Contribution to the topical issue "Electrical Engineering Symposium (SGE 2016)", edited by Adel Razek
}

homogeneously in the rest of the amorphous structure (1-2 nm of amorphous separation of nano-grains). It is more than $70 \%$ of crystalline phase against the rest of the amorphous phase very poor of Si. The formation of such structure with grain size around $10-15 \mathrm{~nm}$ requires a high nucleation rate as well as a slow growth rate of the grains. The nanocrystalline alloys with the composition of $\mathrm{Fe}_{73.5} \mathrm{Cu}_{1} \mathrm{Nb}_{3} \mathrm{Si}_{15.5} \mathrm{~B}_{7}$ (studied materials in this work) owe their nanocrystallization to the 1 at.\% $\mathrm{Cu}$ and 3 at.\% $\mathrm{Nb}$ playing, respectively, the role of nucleation and controlling the grain size $[4,5]$.

Applying the annealing $\left(500-600{ }^{\circ} \mathrm{C}, 1 \mathrm{~h}\right)$ without an external magnetic field allows to obtain nanocrystalline alloys with high DC (direct current) permeability ranged between 500000 and $10^{6}$. This permeability can be fixed down in a controlled way to amplitudes of $10^{4}, 10^{3}$ and even $10^{2}$. Such a wide range of permeability is possible due to the annealing steps: annealing under transversal magnetic field and annealing under mechanical stress $[6,7]$.

The paper deals with the ageing study of a high permeability (HP) nanocrystalline material with the maximum permeability $\mu_{\max }^{1} \mathrm{~Hz}$ around 500000 .

\section{Experiment}

Using the melt spinning process, amorphous ribbons of composition $\mathrm{Fe}_{73.5} \mathrm{Cu}_{1} \mathrm{Nb}_{3} \mathrm{Si}_{15.5} \mathrm{~B}_{7}$ were prepared by rapid 
The European Physical Journal Applied Physics

Table 1. Magnetic property values before ageing treatment for high permeability materials.

\begin{tabular}{|c|c|c|c|c|c|c|c|c|c|}
\hline & & \multicolumn{2}{|c|}{$H_{\mathrm{c}}(\mathrm{A} / \mathrm{m})$} & \multicolumn{2}{|c|}{$\mu_{\mathrm{i}}$} & \multicolumn{2}{|c|}{$P_{\text {hys }}\left(10^{-3} \mathrm{~W} / \mathrm{kg}\right)$} & \multicolumn{2}{|c|}{$P_{\text {dyn }}(\mathrm{W} / \mathrm{kg})$} \\
\hline & & Sample 1 & Sample 2 & Sample 1 & Sample 2 & Sample 1 & Sample 2 & Sample 1 & Sample 2 \\
\hline High & $100{ }^{\circ} \mathrm{C}$ & 0.67 & 0.69 & 205300 & 177900 & 0.43 & 0.45 & 0.47 & 0.47 \\
\hline permeability & $150{ }^{\circ} \mathrm{C}$ & 0.64 & 0.65 & 199100 & 191200 & 0.43 & 0.44 & 0.46 & 0.45 \\
\hline cores & $200{ }^{\circ} \mathrm{C}$ & 0.65 & 0.67 & 183800 & 155000 & 0.44 & 0.45 & 0.45 & 0.47 \\
\hline & $240{ }^{\circ} \mathrm{C}$ & 0.66 & 0.67 & 187900 & 195100 & 0.43 & 0.44 & 0.49 & 0.47 \\
\hline
\end{tabular}

quenching. The ribbons obtained were $\sim 20 \mu \mathrm{m}$ thick and $7 \mathrm{~mm}$ wide. They were wound into toroidal cores of $22 \mathrm{~mm}$ and $17 \mathrm{~mm}$ in external and internal diameters, respectively. In order to obtain the HP cores a conventional annealing without external field at $530{ }^{\circ} \mathrm{C}$ during $1 \mathrm{~h}$ was applied to the as obtained amorphous cores.

The obtained HP cores were subjected to four continuous thermal ageing of $100,150,200$ and $240{ }^{\circ} \mathrm{C}$ (the 200 and $240{ }^{\circ} \mathrm{C}$ temperatures were chosen to accelerate the ageing). The ageing treatments were driven in atmospheric conditions. For each combination (material nuance, aging temperature), there were two identical samples for checking the measurement dispersions. A specific ageing monitoring protocol is followed to determine the evolution of magnetic properties. After each ageing step, the cores were subjected to magnetic measurements. The means used were: hysteresigraph bench for quasi-static and dynamic loops (B-H loops) and impedance analyzer (Agilent $4294 \mathrm{~A}$ ) within a frequency range from $40 \mathrm{~Hz}$ to $10 \mathrm{MHz}$ for complex permeability $\left(\mu^{\prime}, \mu^{\prime \prime}\right)$. In order to follow the evolution of the eddy current losses, resistivity measurements were made using the nanocrystalline ribbons. They were cut carefully from a core into $10 \mathrm{~cm}$ length and subjected to the same thermal ageing treatments as cores were subjected to. The method used is the four points method.

All the following characteristics were measured at room temperature (RT):

- coercive force $H_{\mathrm{c}}$, the saturation flux density $B_{\text {sat }}$ and the hysteresis losses $P_{\text {hys }}$ (quasi-static B-H loops measured at $1 \mathrm{~Hz}$ ),

- the dynamic losses $P_{\text {dyn }}$ (dynamic B-H loops measured at $1 \mathrm{kHz}$ for a sinusoidal imposed flux density of $0.8 \mathrm{~T}$ ),

- initial permeability $\mu_{\mathrm{i}}$ (impedance analyzer: real part $\mu^{\prime}$ of $\mu$ at very low field $(<0.5 \mathrm{~A} / \mathrm{m})$,

- the resistivity $\rho$ (the four points method).

\section{Results}

The magnetic parameters are identified at each ageing step are plotted in relative values calculated from the initial ones (before ageing treatment) using equation (1). The curves are function of ageing time $t_{\mathrm{a}}\left(t_{\mathrm{a} \_\max }=2900 \mathrm{~h}\right)$ and parameterized in ageing temperature $T_{\mathrm{a}}$.

$$
\frac{\Delta M P}{M P}=\frac{M P\left(t_{\mathrm{a}}\right)-M P\left(t_{\mathrm{a}}=0\right)}{M P\left(t_{\mathrm{a}}=0\right)} \times 100 \% .
$$

MP: magnetic property.

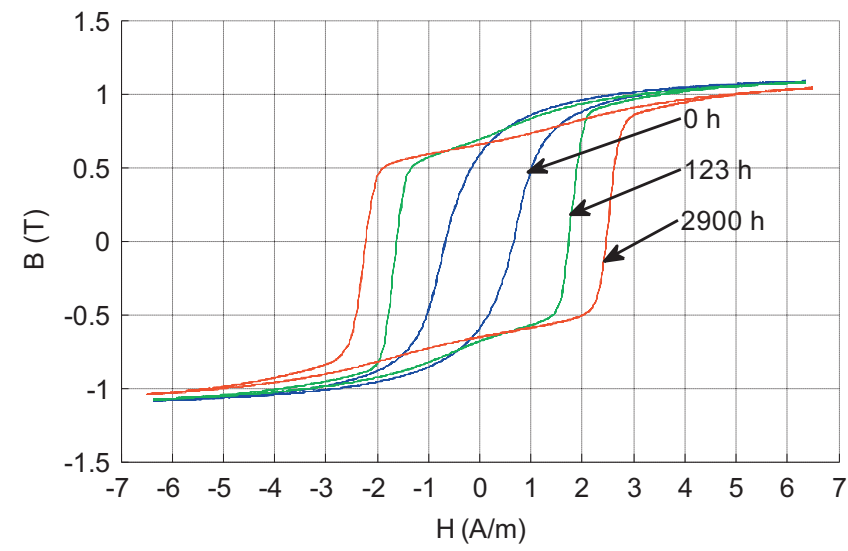

Fig. 1. Quasi-static hysteresis loops of a high permeability core measured at $1 \mathrm{~Hz}$ for different ageing times of the $240{ }^{\circ} \mathrm{C}$ accelerated ageing.

\subsection{Initial state of nanocrystalline cores (before ageing treatment)}

The high permeability cores show very soft magnetic properties. Table 1 shows the values of the coercive force $H_{\mathrm{c}}$, the initial permeability $\mu_{\mathrm{i}}$, the hysteresis losses $P_{\text {hys }}$ and the dynamic losses $P_{\text {dyn }}$ of all HP studied cores. The mean values of the magnetic properties are: $\left\langle H_{\mathrm{c}}\right\rangle=0.42 \mathrm{~A} / \mathrm{m}$, $\left\langle\mu_{\mathrm{i}}\right\rangle=29000,\left\langle P_{\text {hys }}\right\rangle=0.44 \times 10^{-3} \mathrm{~W} / \mathrm{kg}$ and $\left\langle P_{\text {dyn }}\right\rangle=$ $0.47 \mathrm{~W} / \mathrm{kg}$.

\subsection{Quasi-static characterizations}

Figure 1 shows several quasi-static B-H loops for the $240{ }^{\circ} \mathrm{C}$ accelerated ageing of a $\mathrm{HP}$ core at different ageing times $(0 \mathrm{~h}, 123 \mathrm{~h}$ and $2900 \mathrm{~h})$. Measurements have been made at $1 \mathrm{~Hz}$ and at RT.

Depending on the ageing time, the hysteresis loops expand and change their shape. The B-H loops pass quickly from a round edged loop to a square edged loop. Furthermore, the coercive force, the hysteresis losses increase and the maximum permeability decreases with ageing time.

\subsubsection{The coercive force $\mathrm{H}_{\mathrm{c}}$}

Figure 2 shows the $H_{\mathrm{c}}$ evolutions as a function of $t_{\mathrm{a}}$ for the four aging temperatures. The coercive force evolutions 
A. Lekdim et al.: Magnetic properties evolution of a high permeability nanocrystalline

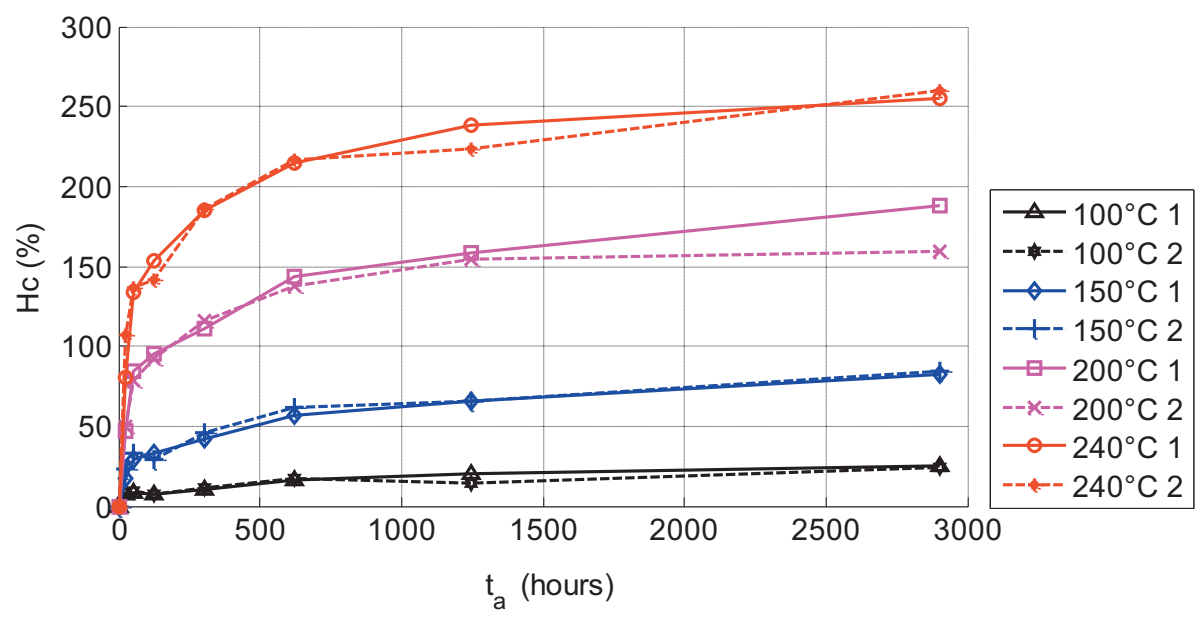

Fig. 2. Evolution of $H_{\mathrm{c}}$ of the high permeability core as a function of $t_{\mathrm{a}}$ for the four ageing temperatures.

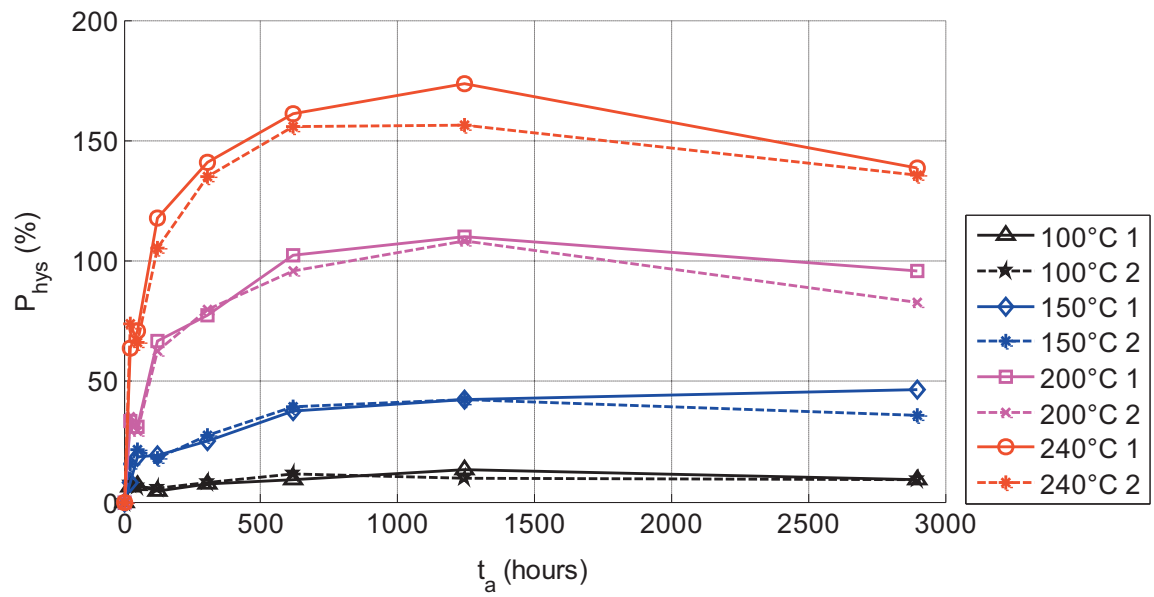

Fig. 3. Evolution of $P_{\text {hys }}$ of the high permeability core as a function of $t_{\mathrm{a}}$ for the four ageing temperatures.

are monotonic and increases with increasing $T_{\mathrm{a}}$. The figure shows a sharp increase at the beginning and then little after $600 \mathrm{~h}$ of $t_{\mathrm{a}} . H_{\mathrm{c}}$ reaches almost $250 \%$ of increase after $2900 \mathrm{~h}$ for the $240{ }^{\circ} \mathrm{C}$ accelerated ageing.

\subsubsection{Hysteresis losses $P_{\text {hys }}$}

Figure 3 shows the evolution of $P_{\text {hys }}$ as a function of ageing time $t_{\mathrm{a}}$ and temperature $T_{\mathrm{a}}$.

Until around $1200 \mathrm{~h}$ of ageing, the evolution of $P_{\text {hys }}$ is monotonic and increases with increasing $T_{\mathrm{a}}$. After that, $P_{\text {hys }}$ decreases a little to reach, respectively, 90 and $160 \%$ of a maximum increase for the 200 and $240{ }^{\circ} \mathrm{C}$ accelerated ageing. For the 100 and $150{ }^{\circ} \mathrm{C}$ ageing treatments, the evolution of $P_{\text {hys }}$ is quasi-stabilized after $1200 \mathrm{~h}$.

\subsubsection{Saturation flux density $B_{\text {sat }}$}

Figure 4 presents a superposition of two hysteresis loops before and after $4300 \mathrm{~h}$ of ageing at $240{ }^{\circ} \mathrm{C}$ measured at
$15 \mathrm{~Hz}$ at RT. Approaching towards the real saturation, $B_{\text {sat }}$ was checked for a relatively high excitation around $100 \mathrm{~A} / \mathrm{m}$. The saturation flux density does not change with ageing (see the zoom in Fig. 4).

\subsection{Impedance-analyzer measurements}

\subsubsection{Initial permeability $\mu_{\mathrm{i}}$}

Figure 5 shows the evolution of $\mu_{\mathrm{i}}$ as a function of $t_{\mathrm{a}}$ and $T_{\mathrm{a}}$. The permeability $\mu_{\mathrm{i}}$ decreases strongly with the increase of $T_{\mathrm{a}}$. The decrease in $\mu_{\mathrm{i}}$ is important at the beginning of the ageing treatment and after about $500 \mathrm{~h}$ it becomes weaker. The maximum reduction is about $-75 \%$ after $2900 \mathrm{~h}$ of ageing at $240{ }^{\circ} \mathrm{C}$.

\subsection{Dynamic characterizations}

Figure 6 shows several dynamic hysteresis loops corresponding to different ageing times $(0 \mathrm{~h}, 123 \mathrm{~h}$ and 


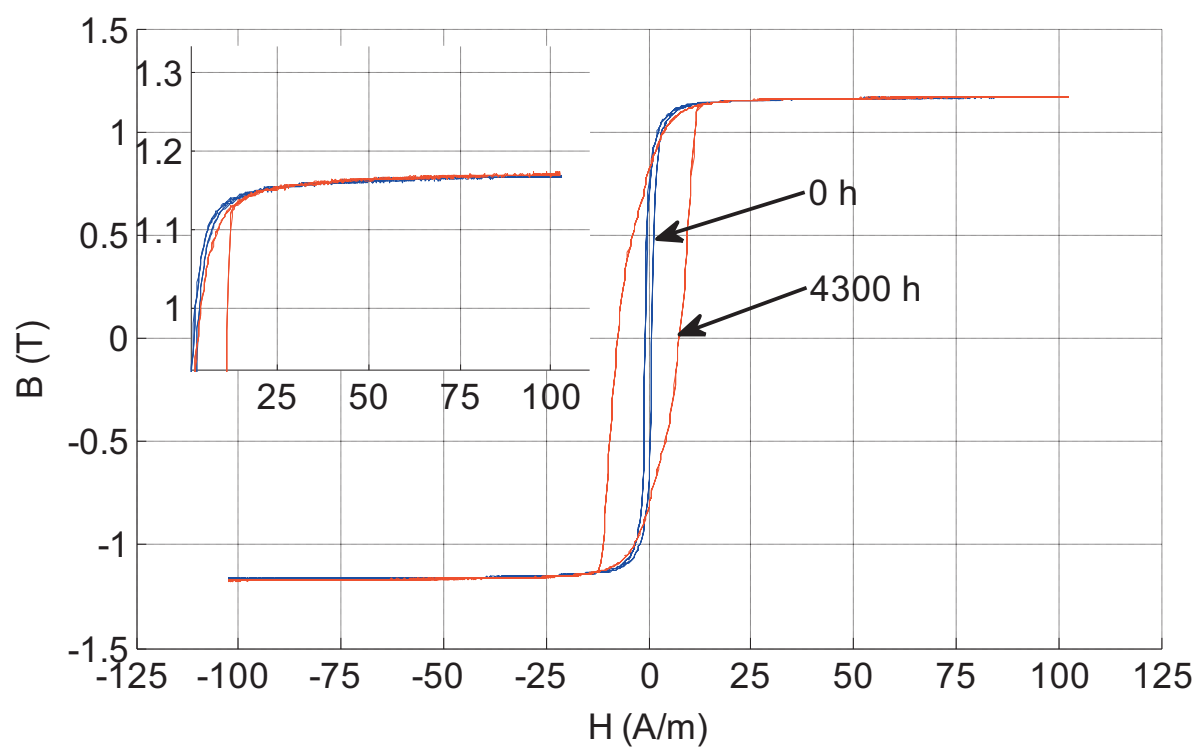

Fig. 4. Hysteresis loops measured at $15 \mathrm{~Hz}$ and RT for before and after $4300 \mathrm{~h}$ of ageing at $240{ }^{\circ} \mathrm{C}$.

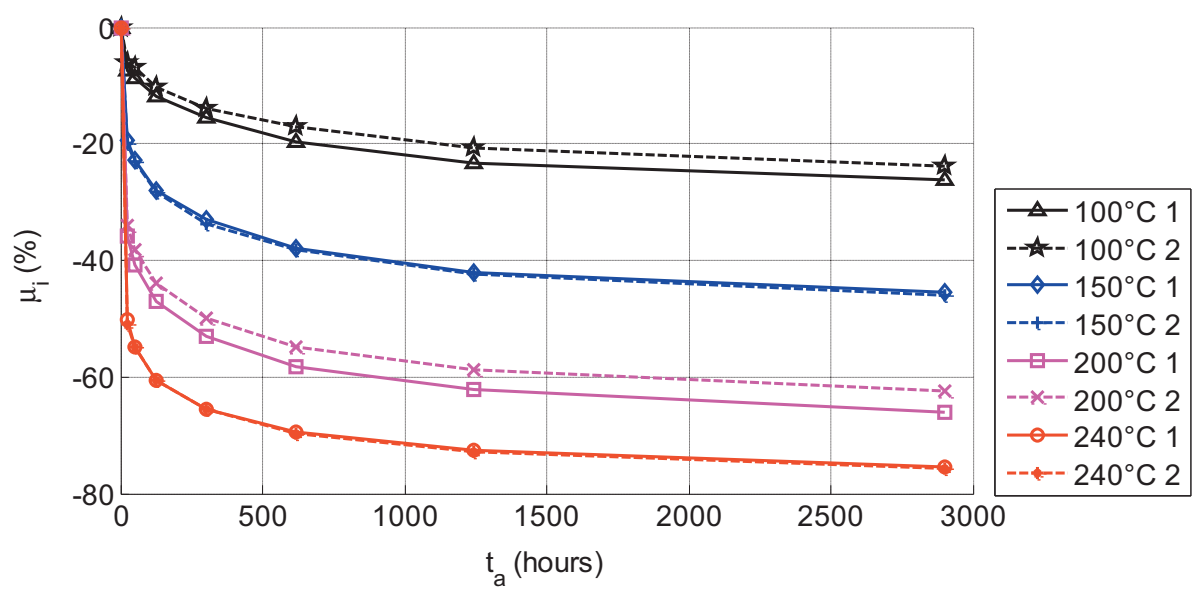

Fig. 5. Evolution of $\mu_{\mathrm{i}}$ of the high permeability core as a function of $t_{\mathrm{a}}$ for the four ageing temperatures.

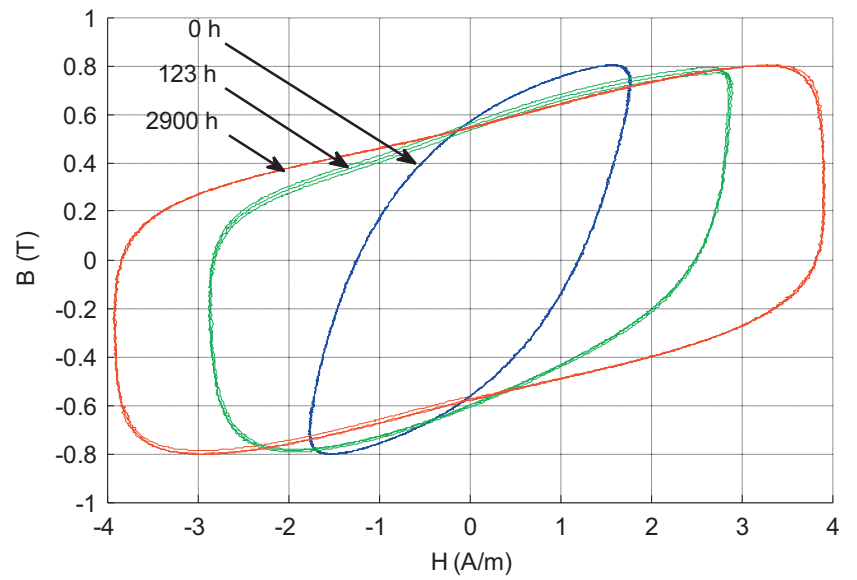

Fig. 6. Dynamic hysteresis loops of the of the high permeability core at $1 \mathrm{kHz} / 0.8 \mathrm{~T}$ for $240{ }^{\circ} \mathrm{C}$ for different ageing times.
$2900 \mathrm{~h}$ ) for the $240{ }^{\circ} \mathrm{C}$ accelerated ageing. They were measured for a maximum sinusoidal induction of $0.8 \mathrm{~T}$ at $1 \mathrm{kHz}$.

As the ageing time increases, the dynamic loops expand and change their shape which causes the rise of the dynamic losses $P_{\text {dyn }}$.

\subsubsection{Dynamic losses (total losses) $\mathrm{P}_{\text {dyn }}$}

Figure 7 shows the evolution of $P_{\mathrm{dyn}}$ as a function of $t_{\mathrm{a}}$ and $T_{\mathrm{a}}$.

The evolutions of the losses $P_{\text {dyn }}$ are monotonic and increase with increasing of the ageing temperature $T_{\mathrm{a}}$. They increase quickly at the beginning and slowly after $1200 \mathrm{~h}$ of ageing time for the four temperatures. For the 100 and $150{ }^{\circ} \mathrm{C}$ ageing treatments, the evolution of $P_{\text {hys }}$ is quasi-stabilized after $1200 \mathrm{~h}$. 
A. Lekdim et al.: Magnetic properties evolution of a high permeability nanocrystalline

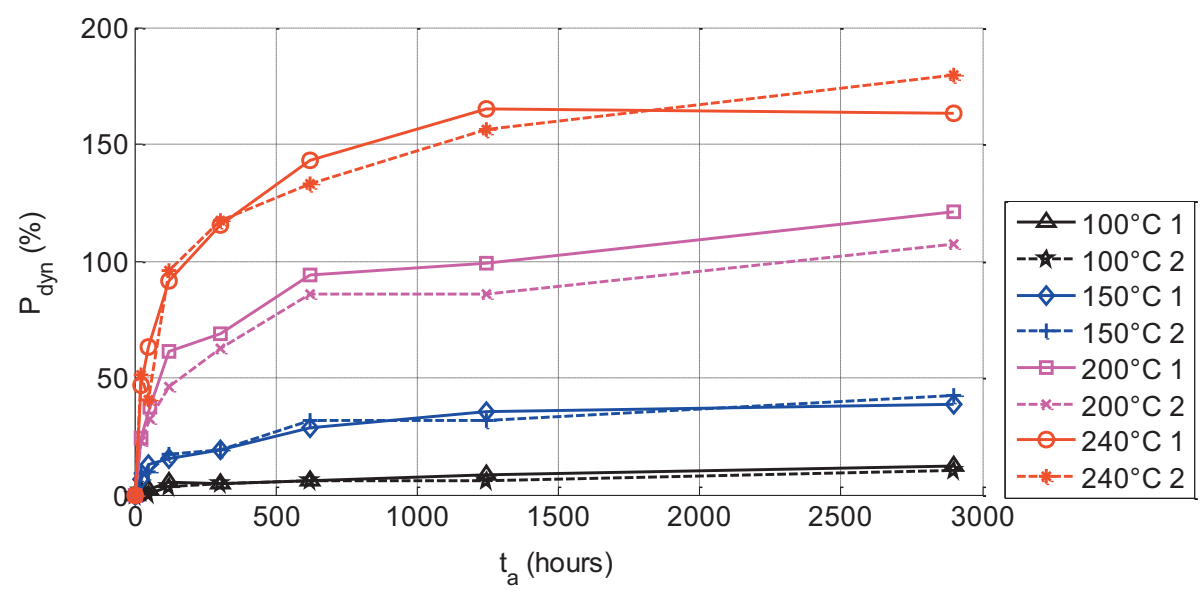

Fig. 7. Evolution of $P_{\mathrm{dyn}}$ of the high permeability core as a function of $t_{\mathrm{a}}$ for the four ageing temperatures.

\subsection{Resistivity measurements}

The four points method is applied on the HP aged ribbons did not show any noticeable evolution of the resistivity $\rho$ during the studied ageing treatments. The resistivity $\rho$ is around $115 \mu \Omega \mathrm{cm}$.

\subsection{Sum-up of the ageing results}

Table 2 presents the relative values (calculated using Eq. (1)) of the properties discussed above for $t_{\mathrm{a} \_\max }=$ $2900 \mathrm{~h}$ and for the four ageing temperatures.

\section{Discussion}

In order to understand and give assumptions to explain the evolutions of the magnetic properties during ageing, an analysis must be done at the different magnetic energies scale that govern the magnetization in these HP nanocrystalline materials.

\subsection{The anisotropy energies}

The total energy developed during the magnetization process is almost equal to the anisotropic energies addition. This energy can be calculated using the initial magnetization curve:

$$
W\left(\frac{J}{m^{3}}\right)=\int_{0}^{B_{\mathrm{sat}}} H \mathrm{~d} B \approx E_{K}=\left\langle E_{K_{1}}\right\rangle+E_{K_{u}}+E_{\lambda \sigma} .
$$

\subsubsection{Definition of the anisotropy energy terms $\left\langle E_{K_{1}}\right\rangle$, $\mathrm{E}_{\mathrm{K}_{\mathrm{u}}}$ and $\mathrm{E}_{\lambda \sigma}$}

The term $\left\langle E_{K_{1}}\right\rangle$ is the average of the random magnetocrystalline anisotropy. The magnetic properties of the crystalline materials like FeSi, FeCo, FeNi, etc. exhibit an average grain size $D$ larger than the exchange
Table 2. Relative evolutions of the principal properties measured at $2900 \mathrm{~h}$ for the four ageing temperatures.

\begin{tabular}{ccccc}
\hline & $100{ }^{\circ} \mathrm{C}$ & $150{ }^{\circ} \mathrm{C}$ & $200{ }^{\circ} \mathrm{C}$ & $240{ }^{\circ} \mathrm{C}$ \\
\hline$H_{\mathrm{c}}(\%)$ & +24 & +84 & +169 & +260 \\
$\mu_{\mathrm{i}}(\%)$ & -25 & -44 & -70 & -80 \\
$B_{\text {sat }}(\%)$ & \pm 0 & \pm 0 & \pm 0 & \pm 0 \\
$P_{\text {hys }}(\%)$ & +9 & +40 & +89 & +135 \\
$P_{\text {tot }}(\%)$ & +10 & +38 & +114 & +170 \\
$\rho(\%)$ & \pm 0 & \pm 0 & \pm 0 & \pm 0 \\
\hline
\end{tabular}

length $l_{\text {ex }}$ (Bloch wall width). This structure allows for the magnetization to follow the easy axis direction of each grain and then magnetic domains can be formed within the grains. The magnetization would, therefore, strongly relate to the magnetocrystalline anisotropy energy $E_{K_{1}}$. Herzer explains with his random anisotropy model developed in 1990 [8,9] that the magnetization in the nanocrystalline materials, due to their structure where the grain size $D$ is almost three times smaller than the exchange length $l_{\mathrm{ex}}$, is no longer sensitive to the magnetocrystalline anisotropy but to its random fluctuations and then to the random magnetocrystalline anisotropic energy $\left\langle E_{K_{1}}\right\rangle$. The latter is significantly smaller than the magnetocrystalline anisotropic energy $E_{K_{1}}$.

The term $E_{K_{u}}$ is the uniform field anisotropic energy. This energy can be induced either under a transversal magnetic field or under longitudinal magnetic field. This energy depends strongly on the chemical composition of the material and the Curie temperature $T_{\mathrm{C}}$. It is induced only under temperatures below $T_{\mathrm{C}}$. The higher $T_{\mathrm{C}}$ is, the more $E_{K_{u}}$ will be induced. The kinetics of its formation also depends on the chemical composition and the annealing temperature $T_{\mathrm{a}}$. It reaches an equilibrium value after an infinite theoretical annealing time. The external field during annealing serves only to align the local magnetization $[5,10]$.

The term $E_{\lambda \sigma}$ is the magnetoelastic anisotropic energy. This energy anisotropy is linked to the saturation magnetostriction constant $\lambda_{\mathrm{s}}$ and to the internal stress $\sigma_{\mathrm{i}}$ which is the result of the mechanical balance between the 


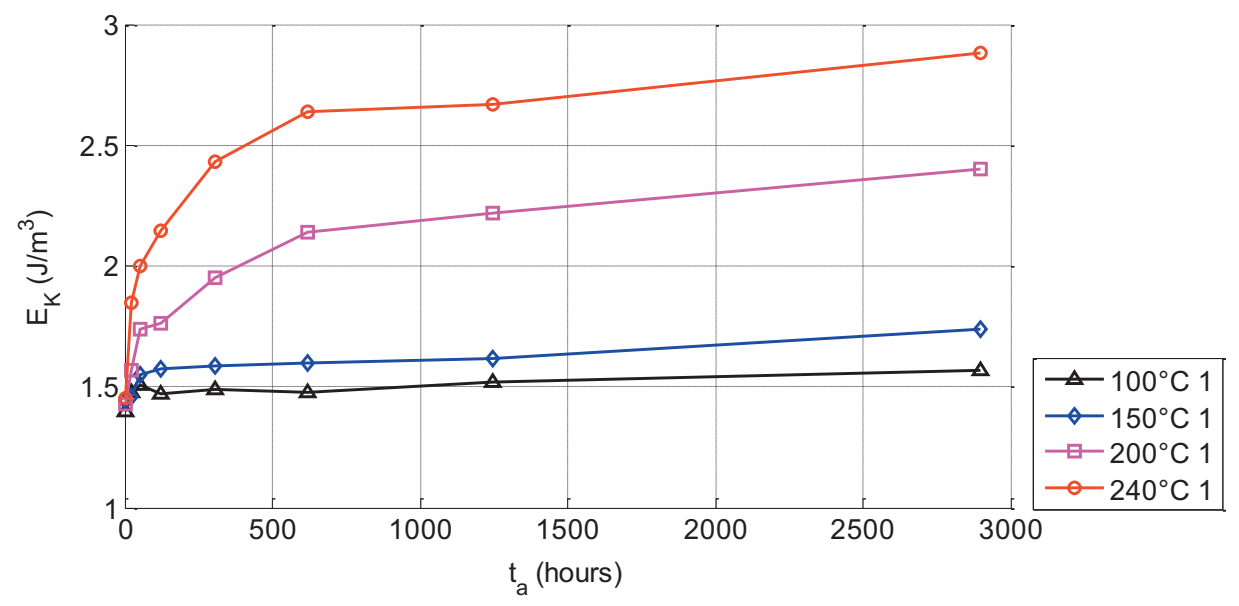

Fig. 8. Evolution of $E_{K}$ as a function of $t_{\mathrm{a}}$ for the four ageing temperatures.

crystalline and the rest of the amorphous phase. The anisotropy $E_{\lambda \sigma}$ can be direct: mechanical deformations occur due to the magnetization process, or inverse: changes in magnetization occur due to external mechanical stress $[6,11,12]$.

\subsubsection{Evolution of the total anisotropy energy $E_{K}$ with ageing}

Figure 8 shows the evolution of $E_{K}$ as a function of $t_{\mathrm{a}}$ for the four ageing temperatures.

The calculation of this anisotropy energy is established by applying the integral of equation (2) to the different first magnetization curves obtained at each ageing step.

The curves of Figure 8 show a strong increment of $E_{K}$ at the beginning of the ageing time especially for the ageing temperatures 200 and $240{ }^{\circ} \mathrm{C}$. This means that the phenomenon related to the increase of $E_{K}$ is thermally activated at earlier stage of ageing treatments. According to equation (2), $E_{K}$ is composed of three terms $\left\langle E_{K_{1}}\right\rangle$, $E_{K_{u}}$ and $E_{\lambda \sigma}$. Therefore, the evolution of $E_{K}$ during ageing may be related to one or more of these three terms. Or, completely, to one or more new anisotropy energies which arise during ageing treatments. A suspicion at the microscopic scale would be on the variation of the volume crystallized fraction $f_{x}$, the variation of the grain size $D$ and the variation of saturation magnetostriction constant $\lambda_{\mathrm{s}}$

\subsubsection{Evolution of the random magnetocrystalline anisotropy energy $\left\langle E_{\mathrm{K}_{1}}\right\rangle$ with ageing}

The dependency of $\left\langle E_{K_{1}}\right\rangle$ on the grain size $D$ is conditioned by the dominance of the anisotropy energies in the material. For example, when $E_{K_{u}}$ dominates over $\left\langle E_{K_{1}}\right\rangle$ in the case of material annealed under transverse field, the expression of $\left\langle E_{K_{1}}\right\rangle$ changes from a dependence of $D^{6}-D^{3}[13,14]$. For the studied nanocrystalline cores (which have undergone an annealing without external field), the dominant anisotropy energy is $\left\langle E_{K_{1}}\right\rangle$ and thus follows the law $D^{6}$ :

$$
\left\langle E_{K_{1}}\right\rangle=f_{x}^{2} \frac{K_{1}^{4} D^{6}}{A^{3}} .
$$

The anisotropy $\left\langle E_{K_{1}}\right\rangle$ depends also of the magnetocrystalline anisotropy constant $K_{1}$, the exchange constant $A$ and the crystallized fraction $f_{x}$. In order to evaluate the variation of $\left\langle E_{K_{1}}\right\rangle$ as a function of ageing it is necessary to evaluate the variation of each parameter $\left(D, f_{x}, K_{1}\right.$ and $A)$ as a function of ageing.

\subsubsection{Variation of the crystallized fraction $f_{x}$ and the gain size $D$}

The variation of $D$ is linked to the variation of $f_{x}$ in this material. In order to check this variation, measurements of X-ray diffraction (XRD) were made on the studied nanocrystalline material. Figure 9 shows two XRD diffractograms for before and after the $240{ }^{\circ} \mathrm{C}$ accelerate ageing of the HP material.

No new peak did appear during ageing at $240{ }^{\circ} \mathrm{C}$ and also at lower ageing temperatures (100, 150 and $200{ }^{\circ} \mathrm{C}$ ). Therefore, the appearance of a new phase is excluded. The high peak corresponds to the crystal plane (110) and the small peak corresponds to the crystal plane (200). This result is confirmed by the resistivity and the TEP (thermo-electrical power) measurements which do not exhibit any variation during the ageing treatments.

The calculation of the width at half height $I(1 / 2)$ of the peak (110) before and after ageing gives information about the evolution of crystalline domains (which corresponds to the grain size $D$ in the case of the studied materials) and, consequently, about the evolution of the crystallized fraction $f_{x}$. Figure 10 shows two histograms indicating the width $I(1 / 2)$ before and after $1320 \mathrm{~h}$ of the $240{ }^{\circ} \mathrm{C}$ accelerated ageing.

After the ageing, the width $I(1 / 2)$ is increased of about $2 \%$. According to the Scherrer formula explained in $[15,16]$, the increase in the width $I(1 / 2)$ would tend to 
A. Lekdim et al.: Magnetic properties evolution of a high permeability nanocrystalline

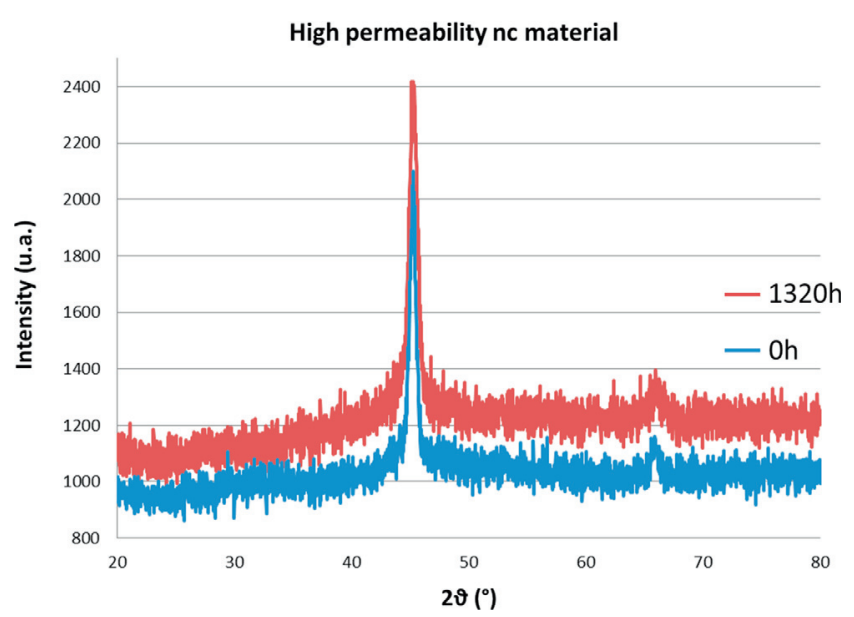

Fig. 9. XRD diffractograms before and after the $240{ }^{\circ} \mathrm{C}$ accelerated ageing of the high permeability nanocrystalline material.

a reduction of the average grain size. This does not make sense, because the aging treatment applied in this study would be favorable to an increase in the average grain size by atomic diffusion of the rest of silicon content from the amorphous phase to the crystalline phase (the applied ageing treatment is comparable to long time application of a low temperature annealing). This increase of $I(1 / 2)$ would be related to the measurement uncertainty of the apparatus.

Therefore, the grain size $D$ and the fraction $f_{x}$ have not changed during ageing treatments.

\subsubsection{Variation of the magnetocrystalline anisotropy constant $K_{1}$}

The constant $K_{1}$ is intrinsic to the FeSi crystals and its value depends on the measurement temperature and the chemical composition. Since the chemical composition has not changed as a function of ageing (no change at the peaks of XRD measurements) and the magnetic characterizations were made at room temperature, the constant $K_{1}$ can be considered unchanged.

\subsubsection{Variation of the exchange constant $A$}

The exchange constant $A$ varies with the saturation flux density $B_{\text {sat }}$ as following [5]:

$$
\frac{A(T)}{A(0 K)}=\left[\frac{B_{\text {sat }}(T)}{B_{\text {sat }}(0 K)}\right]^{2} \text {. }
$$

For the studied nanocrystalline material, the saturation flux density did not change with ageing treatments (as shown in Sect. 3.2). So, according to equation (4), the exchange constant $A$ has not changed as a function of ageing treatments.

In conclusion, the random anisotropy energy $\left\langle E_{K_{1}}\right\rangle$ has not changed with ageing treatments.

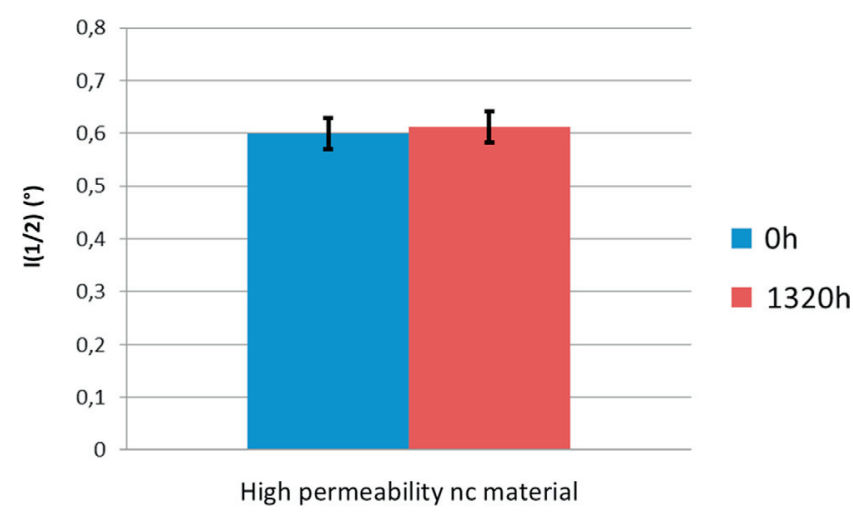

Fig. 10. Changing in the half height width of the peak (110) by the $240{ }^{\circ} \mathrm{C}$ accelerated ageing treatment for the high permeability nanocrystalline material.

\subsubsection{Evolution of the magnetoelastic anisotropy energy} $\mathrm{E}_{\lambda \sigma}$ with ageing

Herzer demonstrates in [17] that for a nanocrystalline chemical composition containing around 15 at.\% of $\mathrm{Si}$ undergoing an annealing without field at $540{ }^{\circ} \mathrm{C}$ during $1 \mathrm{~h}$ allows to reach zero-magnetostriction. Before ageing treatments, the measurement of $\lambda_{\mathrm{s}}$ using the SAMR (small angle magnetization rotation) bench [18] gives $\lambda_{\mathrm{s}}$ almost equal to zero $\left(\lambda_{\mathrm{s}} \approx 0.15 \mathrm{ppm}\right)$ which confirms the Herzer's demonstration.

During ageing, measurements of $\lambda_{\mathrm{s}}$ were made on aged flat ribbons of the HP nanocrystalline material using the SAMR bench. After around $2100 \mathrm{~h}$ of ageing at $240{ }^{\circ} \mathrm{C}$, $\lambda_{\mathrm{s}}$ has decreased to reach $0.087 \mathrm{ppm}$ i.e., a relative decrease of $42 \%$. The anisotropy energy $E_{\lambda \sigma}$ depends on $\lambda_{\mathrm{s}}$ as following:

$$
E_{\lambda \sigma}\left(\frac{J}{m^{3}}\right) \approx-\frac{3}{2} \lambda_{\mathrm{s}} \sigma_{\mathrm{i}} .
$$

The constant $\sigma_{\mathrm{i}}$ corresponds to the internal stress. The ageing treatments applied to the samples have not been made under mechanical stress, therefore, there would be a small probability for changing $\sigma_{\mathrm{i}}$ (no back stress effect $[6,17])$. Assuming that the internal stress $\sigma_{\mathrm{i}}$ did not vary with aging, the change in $E_{\lambda \sigma}$ anisotropy would be proportional to that of $\lambda_{\mathrm{s}}$ (according to Eq. (5)). Thus, before ageing $E_{\lambda \sigma}$ was small and with ageing it becomes more and more small.

\subsubsection{Evolution of the uniform anisotropy energy $E_{K_{u}}$ with ageing}

The uniform anisotropy $K_{u}$ is induced, basically, by a spontaneous magnetization within each magnetic domain. Thus, the induction of $K_{u}$ is exclusively managed by the local orientations of the magnetic domains $[2,5]$. Since there is no external magnetic field during annealing of HP cores, the magnetic domains are formed with complete random orientations and then the induced $K_{u}$ is 


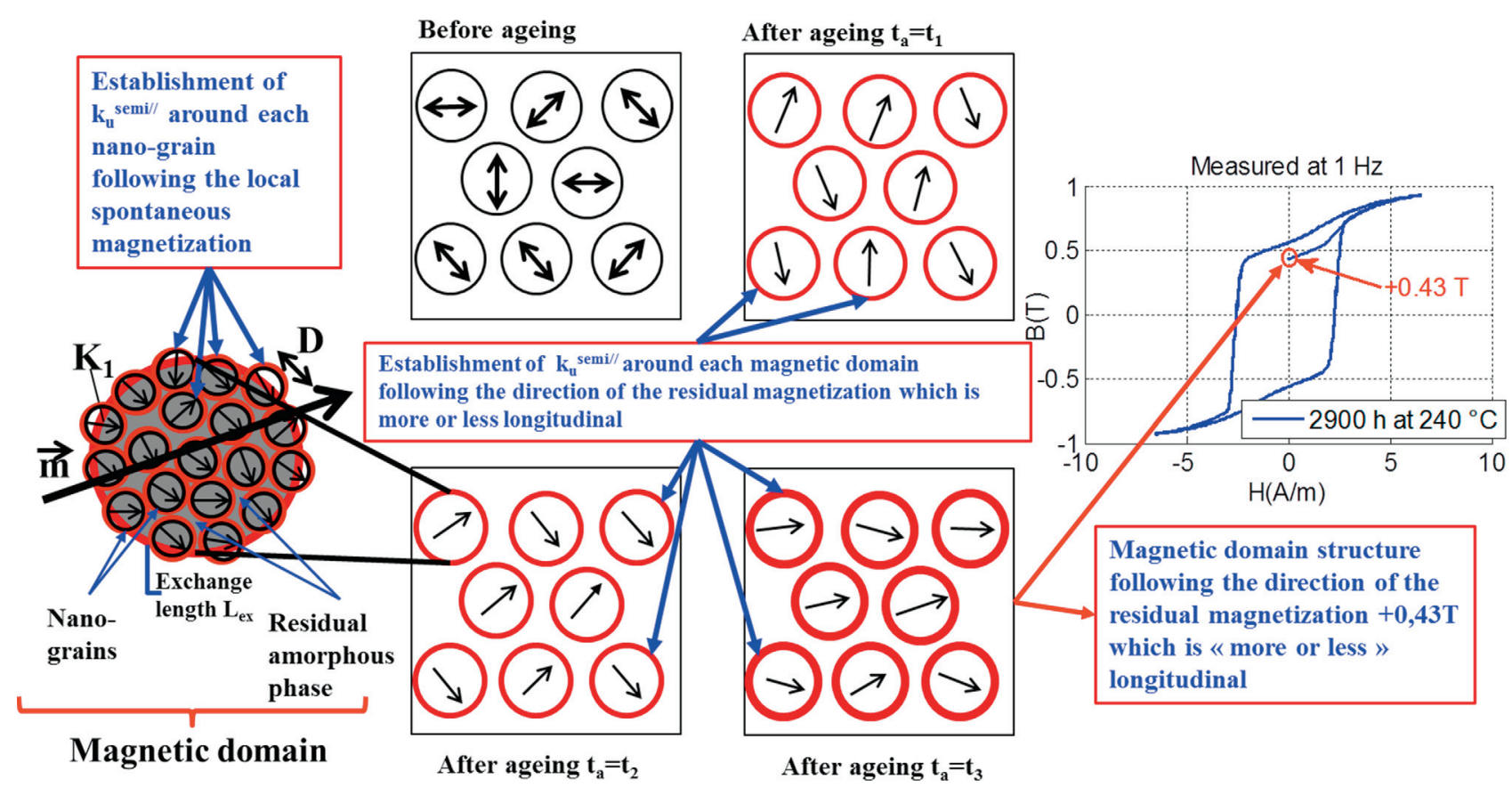

Fig. 11. Illustrative diagram of the evolution of the magnetic domain structure and the induction of $K_{u}^{\text {semi// }}$ during ageing. The quasi-static hysteresis loop of a HP material is measured at $1 \mathrm{~Hz}$ after $2900 \mathrm{~h}$ of ageing at $240{ }^{\circ} \mathrm{C}$.

"incoherent" and "non-uniform" at a macroscopic scale. Furthermore, $1 \mathrm{~h}$ of annealing at $540{ }^{\circ} \mathrm{C}$ is not enough to induce sufficient quantity of the "incoherent" $K_{u}$ to be competitive compared to $\left\langle K_{1}\right\rangle$. Thus, before ageing treatments, the anisotropy energy $E_{K_{u}}$ is neglected whatever its form (coherent or incoherent).

Many hours under temperature (less than the $T_{\mathrm{C}}$ ), promote the establishing of the uniform anisotropy $k_{u}$. If the magnetic domain structure keeps the complete disorientation of its magnetic moments, the induced $K_{u}$ will be "incoherent". But, if the magnetic domains are aligned in a given direction (i.e., with magnetic field or axial mechanical stress) the induced $K_{u}$ will be "coherent" and then uniform at a macroscopic scale.

\subsubsection{Establishment of a new magnetic anisotropy energy with ageing}

In the case of the studied ageing, the last magnetic characterization is done at an imposed induction of $0.8 \mathrm{~T}$ before returning the samples to furnaces. Thus, the HP samples age with a high residual magnetization $B \approx 0.43 \mathrm{~T}$ (as shown in Fig. 11). The domain structure will mainly consist of magnetic domains oriented "more or less" along the longitudinal direction of the nanocrystalline ribbons. So the anisotropy $K_{u}$ will be induced along these directions that are "more or less" longitudinal. In this case the induced $K_{u}$ will not be completely "incoherent" but "semi-coherent" or "semi-uniform" at the macroscopic scale. We note this anisotropy constant $K_{u}^{\text {semi// }}$ and correspondingly the anisotropy energy $E_{K_{u}}^{\text {semi// }}$. This explains the almost rectangular shape of the hysteresis loops.

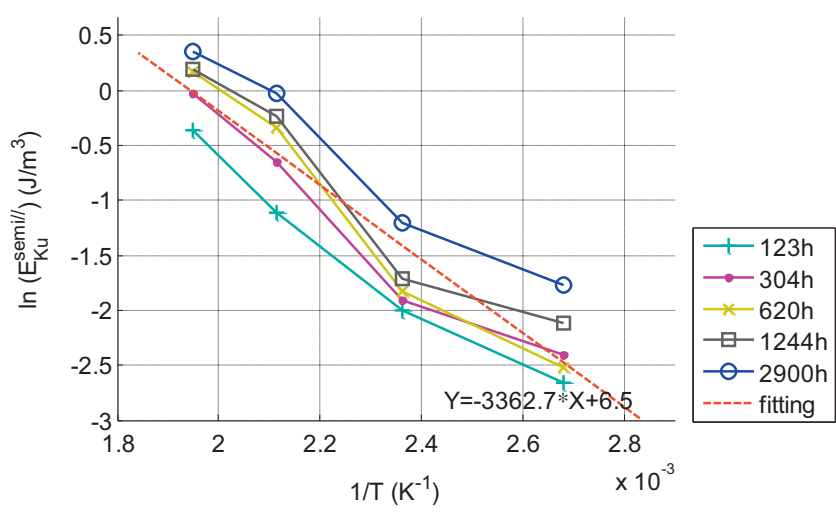

Fig. 12. Presentation of the $\ln K_{u}^{\text {semi// }}$ evolution according to the Arrhenius diffusion model.

Indeed, the applied ageing with a high residual magnetization can be compared to a "non-controlled" annealing under a longitudinal magnetic field.

Figure 11 illustrates the evolution of the magnetic structure and the establishment of the anisotropy $K_{u}^{\text {semi// }}$ as function of ageing time. It shows, also, a quasi-static hysteresis loop of a $\mathrm{HP}$ material measured at $1 \mathrm{~Hz}$ after $2900 \mathrm{~h}$ of ageing at $240{ }^{\circ} \mathrm{C}$. The latter displays a residual magnetization around $+0.43 \mathrm{~T}$.

As a function of ageing, $\left\langle E_{K_{1}}\right\rangle$ remains constant and $E_{\lambda \sigma}$ decreases which, normally, promotes an improvement of the magnetic properties. However, magnetic characterizations show the opposite with degradation of almost all the magnetic properties (losses, coercive field, permeability, ...). The latter is explained by the fact that the constancy of $\left\langle E_{K_{1}}\right\rangle$ and the decrease of $E_{\lambda \sigma}$ with ageing are 


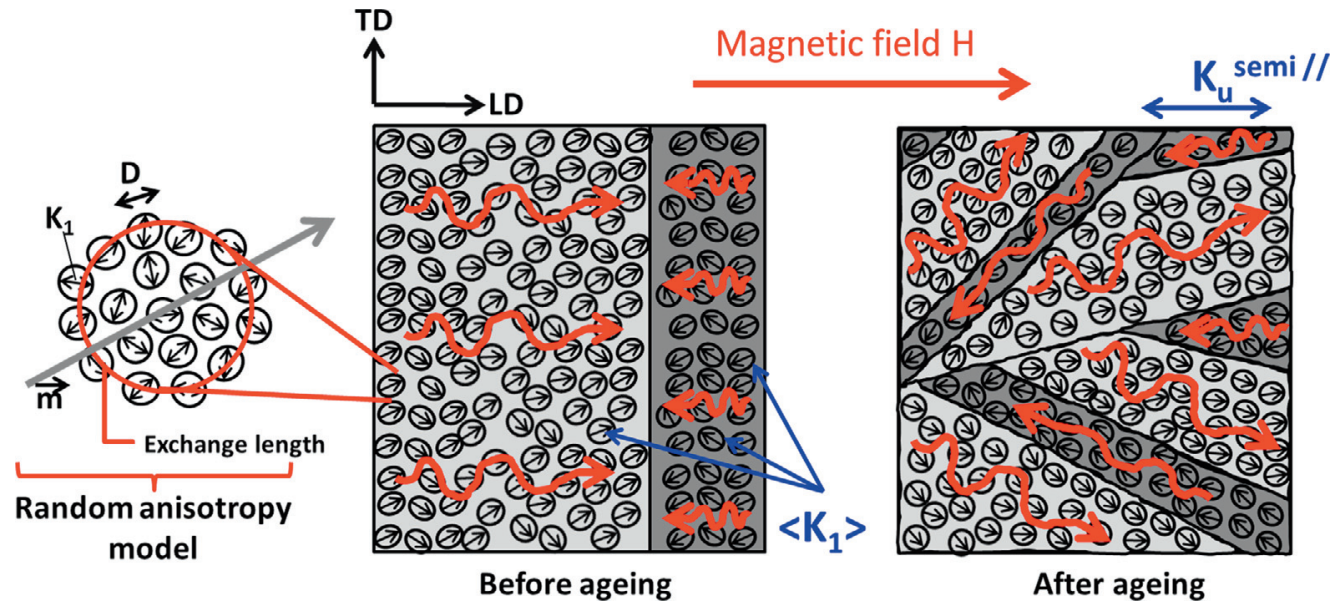

Fig. 13. Illustrative diagram of the random anisotropy model and the influence of the induced anisotropy $K_{u}^{\text {semi// }}$ on the Bloch wall displacement during ageing.

largely compensated by the establishment and the increase of the "semi-coherent" anisotropy energy $\mathrm{E}_{K_{u}}^{\mathrm{semi} / /}$ as explained previously. Therefore, equation (2) becomes:

$$
E_{K}\left(t_{\mathrm{a}}, T_{\mathrm{a}}\right)=\left\langle E_{K_{1}}\right\rangle+E_{\lambda \sigma}\left(t_{\mathrm{a}}, T_{\mathrm{a}}\right)+E_{K_{u}}^{\mathrm{semi} / /}\left(t_{\mathrm{a}}, T_{\mathrm{a}}\right),
$$

where $t_{\mathrm{a}}$ and $T_{\mathrm{a}}$ are respectively time and temperature of ageing.

\subsubsection{Origin of the anisotropy energy $E_{\mathrm{K}_{\mathrm{u}}}^{\mathrm{semi} / /}$}

According to the pair ordering mechanism, pairs of atoms of different kinds interact with the local spontaneous magnetization through energy. The latter, is dependent on the angle between the position of the pairs of atoms and the direction of the local magnetization. It is also dependent of the nature of the pairs of atoms (chemical composition).

For the studied material (FeCuNbSiB), several kinds of pair ordering can be formed between the metal and metalloid atoms (Fe-Si, Fe-B, ...), or between the different metal atoms ( $\mathrm{Fe}-\mathrm{Fe}, \mathrm{Fe}-\mathrm{Cu}, \mathrm{Cu}-\mathrm{Cu}, \ldots$. . ). If any annealing process exhibits sufficient temperature and time, the atoms will diffuse to adopt a structure which distributes the pairs of atoms in the most advantageous manner, i.e., to minimize the resultant energy between the pairs of atoms and the local spontaneous magnetization. In the studied case, the ageing process activates the diffusion of the pairs of atoms to optimize the orientation of the magnetic structure. The latter, results in the induction of the "semi-coherent" anisotropy energy $E_{K_{u}}^{\mathrm{semi} / /}$ in the direction of the residual magnetization which is "more or less" longitudinal.

During the annealing process of the studied HP nanocrystalline materials $\left(\sim 540{ }^{\circ} \mathrm{C}, \sim 1 \mathrm{~h}\right)$, the pair ordering originates from the short range atomic diffusion $[2,5,19]$. Thus, during ageing treatment, the diffusion cannot be long range because the activation energy is lower than that of the annealing process. So, the induction of the anisotropy energy $E_{K_{u}}^{\text {semi// }}$ is related to the short range atomic diffusion. Figure 12 shows the plots of $\ln \left(E_{K_{u}}^{\mathrm{semi} / /}\right)$ as a function of $1 / T_{\mathrm{a}}$ for several ageing times.

The curves of Figure 12 can be approximated to straight lines. Therefore, the short range diffusion follows well the diffusion mechanism according to Arrhenius expressed by:

$$
\ln \left(E_{K_{u}}^{\mathrm{semi} / /}\right)=-\left(\frac{Q}{R}\right) \frac{1}{T_{\mathrm{a}}}+\ln \left(\left[E_{K_{u}}^{\mathrm{semi} / /}\right]_{0}\right),
$$

where $Q$ is the activation energy, $R$ is the Boltzmann constant $(8.32 \mathrm{~J} / \mathrm{K})$ and $\left[E_{K}^{\mathrm{loc}}\right]_{0}$ is the diffusion coefficient when $T_{\mathrm{a}}$ tends to the infinite. From equation (6) and the fitting $(Y=-3362.7 \times X+6.5)$ the identification of $Q$ and $\left[E_{K}^{\text {loc }}\right]_{0}$ gives, respectively, the values: $28000 \pm 2500 \mathrm{~J} / \mathrm{m}^{3}$ and $690 \pm 3 \mathrm{~J} / \mathrm{m}^{3}$.

\subsection{Impact of $E_{K_{u}}^{s e m i / /}$ evolution on magnetic properties as a function of ageing}

The ageing treatments have damaged practically all the magnetic properties namely: $H_{\mathrm{c}}, \mu_{\mathrm{i}}, P_{\text {hys }}$ and $P_{\mathrm{dyn}}$. The reason is related to the fact that $E_{K_{u}}^{\mathrm{semi} / /}$ increases and becomes rapidly competitive to $\left\langle E_{K_{1}}\right\rangle$ and ends by dominating on all the material. As illustrated by Figure 11, $K_{u}^{\text {semi// }}$ is introduced around each magnetic domain and imposes during ageing a domain structure following the direction of the high residual magnetization $(B \sim 0.43)$. During the magnetization process, the obtained structure prevents the formation of large domains (Bloch wall displacement mechanism). Figure 13 illustrates the random anisotropy model and the influence of the induced anisotropy $K_{u}^{\mathrm{semi} / /}$ during ageing on the Bloch wall displacement (formation of the "large" domains).

Indeed, before ageing, the magnetic structure exhibits the formation of one large magnetic domain in the direction of the applied field. After ageing, the magnetic structure exhibits the formation of many small magnetic domains in the direction of the applied field. 
4.2.1 Evolution of the magnetic losses as a function of ageing

According to the reference [20], the magnetic losses decomposition is expressed as following:

$$
P_{\text {tot }}=P_{\text {hys }}+P_{\text {class }}+P_{\text {exc }},
$$

where $P_{\text {tot }}$ is the total losses (assumed to be equal to $\left.P_{\text {dyn }}\right), P_{\text {hys }}$ is the static losses (at low frequencies), $P_{\text {class }}$ is the classical losses (eddy current losses) and $P_{\text {exc }}$ is the excess losses.

Since the resistivity does not vary during the ageing treatments, the classical losses can be considered unchanged during ageing. So the increase in the total (dynamic) losses as a function of ageing is due to:

- the increase of the static losses $P_{\text {hys }}$. Indeed, the losses $P_{\text {hys }}$ are due the Bloch wall interaction with obstacles while moving (the obstacles can be: dislocations, large interstitial atoms, surface defects, grain boundaries, precipitates, etc.). For very low frequencies (necessary condition to calculate $P_{\text {hys }}$ ), the Bloch wall displacement is slow enough to avoid the local induced currents. The ageing has amplified the Bloch wall/

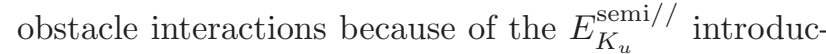
tion around each magnetic domain,

- the large increase of the excess losses $P_{\text {exc }}$. They are related to the same Bloch wall/obstacle interactions which, with frequency, increase due to the creation of local harmonic currents.

\section{Conclusion}

The study of the thermal aging of a high permeability nanocrystalline material was carried out. An analysis of the evolution of the different anisotropy energies as well as the use of XRD results revealed correlations between macroscopic magnetic properties and physical phenomena related to ageing. Thus, the ageing of the high permeability nanocrystalline materials is not linked to the formation of a new precipitates phase $\left(\mathrm{Fe}_{2} \mathrm{~B}, \mathrm{Fe}_{2} \mathrm{O}_{3}, \ldots\right)$ or to the nano-grains growth. It is rather linked to a short range atomic diffusion which is at the origin of "semi-coherent" anisotropy introduction. The latter prevents the Bloch wall displacement and the magnetic domains rotation.

The induction of the anisotropy during ageing treatment is exclusively dependent on the magnetic state of the nanocrystalline material. The present study of ageing was carried out without controlling the magnetization of the samples. However, it is very interesting to carry out other ageing studies but with controlling the magnetization of the samples while ageing. For example, apply demagnetization on the nanocrystalline samples after each characterization step.

The authors thank APERAM Alloys Amilly society for the supply of the NANOPHY ${ }^{\circledR}$ nanocrystalline samples. They, also, thank the laboratory MATEIS for XRD measurements and the laboratory SATIE for the magnetostriction measurements. This study goes within the GENOME (Corac) project.

\section{References}

1. C. Martin, R. Robutel, C. Buttay, F. Sixdenier, High temperature ageing of Fe-based nanocrystalline ribbons, in IMAPS International Conference on HiTEC, Albuquerque, USA, 2012, p. 6

2. T. Waeckerlé, A. Demier, S. Camus, Effect of aging on FeCuNbSiB nanocrystalline magnetic properties, in Soft Magnetic Materials Conference, São Paulo, Brazil, 2015, p. 4

3. Y. Yoshizawa, S. Oguma, K. Yamauchi, J. Appl. Phys. 64, 6044 (1988)

4. G. Herzer, Acta Mater. 61, 718 (2013)

5. R. Hilzinger, W. Rodewald, Magnetic Materials, (Vacuumschmelze GmbH \& Co, Germany, 2013)

6. T. Waeckerle, T. Save, A. Demier, J. Magn. Magn. Mater. 320, e797 (2008)

7. T. Waeckerlé, P. Vérin, P. Cremer, D. Gautard, J. Magn. Magn. Mater. 215, 449 (2000)

8. G. Herzer, IEEE Trans. Magn. 26, 1397 (1990)

9. G. Herzer, IEEE Trans. Magn. 25, 3327 (1989)

10. M. Emura, A.M. Severino, A.D. Santos, F.P. Missell, IEEE Trans. Magn. 30, 4785 (1994)

11. B. Hofmann, H. Kronmüller, J. Magn. Magn. Mater. 152, 91 (1996)

12. F. Alves, J.B. Desmoulins, D. Hérisson, J.F. Rialland, F. Costa, J. Magn. Magn. Mater. 215, 387 (2000)

13. K. Suzuki, G. Herzer, Scr. Mater. 67, 548 (2012)

14. K. Suzuki, N. Ito, S. Saranu, U. Herr, A. Michels, J.S. Garitaonandia, J. Appl. Phys. 103, 07E730 (2008)

15. A.T. D'Agostino, Anal. Chim. Acta 262, 269 (1992)

16. V. Uvarov, I. Popov, Mater. Charact. 58, 883 (2007)

17. G. Herzer, IEEE Trans. Magn. 30, 4800 (1994)

18. F. Alves, P. Houée, M. Lécrivain, F. Mazaleyrat, J. Appl. Phys. 81, 4322 (1997)

19. L.J. Maksymowicz, L. Dargel, M. Lubecka, M. Pyka, J. Magn. Magn. Mater. 35, 281 (1983)

20. G. Bertotti, J. Magn. Magn. Mater. 320, 2436 (2008) 